\title{
Politicas públicas em oncologia: refletindo sobre a atuação da terapia ocupacional
}

\section{Public policies in oncology: reflecting on the role of occupational therapy}

\author{
Ana C. Cardoso da Silva ${ }^{1}$, Andréa Rizzo dos S. Boettger Giardinetto ${ }^{2}$
}

SILVA, A. C. C; GIUARDINETTO, A. R. S. B. Politicas públicas em oncologia: refletindo sobre a atuação da terapia ocupacional. Rev. Ter. Ocup. Univ. São Paulo, v. 23, n. 3, p. 297-308, set./ dez. 2012.

RESUMO: A Política Nacional de Atenção Oncológica almeja o acesso e atendimento integral além de uma maior efetividade e eficiência no controle do câncer. Assim, diversas ações, serviços e profissionais são exigidos para dar a assistência necessária. A respeito da atuação da Terapia Ocupacional, nota-se que a profissão não é especificada, e não tem a atuação regulamentada. Sendo assim, o objetivo principal do presente estudo foi identificar a inserção, atuação e ampliação do campo de trabalho da Terapia Ocupacional no Estado de São Paulo para a área oncológica em contexto hospitalar, tomando por base as Políticas Públicas de Atenção Oncológica. Visou também identificar os Centros de Assistência de Alta Complexidade em Oncologia do Estado de São Paulo que possuem o terapeuta ocupacional como parte da equipe. Para tanto, foi realizada: identificação dos Centros de Alta Complexidade em Oncologia do Estado de São Paulo cadastrados no Cadastro Nacional de Estabelecimentos de Saúde, por meio de dados fornecidos pelo INCA e do site oficial do Ministério da Saúde; mapeamento da inserção do terapeuta ocupacional nesses locais e aplicação de questionário aos profissionais, para caracterizar os serviços e ações desenvolvidas pela Terapia Ocupacional em oncologia. O estudo do tipo qualitativo descritivo constatou a existência de diferentes possibilidades assistenciais em oncologia, tendo em vista as demandas e organização dos hospitais. Quanto à inserção e ampliação do campo de trabalho, verificou-se que nem todos os CACONs contam com os serviços e ações da Terapia Ocupacional, o que sugere que ainda não há efetivamente a incorporação desse profissional nas equipes multiprofissionais desses centros.

DESCRITORES: Oncologia/recursos humanos; Políticas públicas de saúde; Terapia ocupacional; Serviço hospitalar de oncologia/recursos humanos.

\footnotetext{
* Este trabalho é parte do Trabalho de Conclusão de Curso que foi apresentado à Faculdade de Filosofia e Ciências da UNESP - Campus de Marília para conclusão do curso de Terapia Ocupacional, em dezembro de 2011.

1. Terapeuta Ocupacional formada pela UNESP - Campus de Marília. Aprimorada pelo Programa de Aprimoramento Profissional em Terapia Ocupacional Hospitalar pelo Hospital das Clínicas da Faculdade de Medicina de Ribeirão Preto da Universidade de São Paulo.

2. Terapeuta Ocupacional, Doutora em Educação, Docente do curso de Terapia Ocupacional, Departamento Fisioterapia e Terapia Ocupacional da UNESP - Campus de Marília.

Endereços para Correspondência: Ana Cristina Cardoso da Silva. Rua Cícero Soares Ribeiro, 405 Bairro: Vila Carmem São Carlos-SP. CEP: 13575. E-mail: ana_cristinacardoso@hotmail.com
} 


\section{INTRODUÇÃO}

ntes da criação do Sistema Único de Saúde
(SUS), a saúde não era considerada um
direito social. O modelo médico-assistencial privatista adotado dividia os brasileiros em três categorias: os que podiam pagar por serviços de saúde privados; os que tinham direito à saúde pública por serem segurados pela previdência social e os que não possuíam direito algum (BRASIL, 2007).

Diante da desarticulação dos serviços de saúde e dos prejuízos sofridos pela população, despertou-se na sociedade e, principalmente, nos profissionais da saúde e sanitaristas, o desejo de implementar uma reforma sanitária, a fim de transformar o modelo vigente de atenção à saúde. O processo de construção do novo sistema de saúde descentralizado e universal iniciou na década de 80 e, mediante intensas discussões, houve significativo avanço com a promulgação da Constituição Federal e criação do SUS em 1988. No ano de 1990, a regulação do SUS, quanto ao seu funcionamento e organização, se completa com a edição das Leis Orgânicas da Saúde (Lei n ${ }^{\circ}$ 8.080/90 e Lei $\mathrm{n}^{\circ}$ 8.142/90) (BRASIL, 2002).

Atualmente o SUS é considerado um dos maiores sistemas públicos de saúde do mundo e abrange desde o atendimento ambulatorial até o transplante de órgãos, garantindo acesso integral, universal e gratuito para toda a população brasileira (BRASIL, 2006).

Há uma vasta publicação de leis, normas e portarias voltadas a regulamentação das ações em saúde. Referente às possibilidades de incorporação da Terapia Ocupacional no SUS e de sua inserção nas Redes de Atenção a Saúde, identificam-se algumas portarias que regulamentam ou fazem referência à atuação desta profissão (BEIRÃO; ALVES, 2010).

Segundo estudo realizado por Beirão e Alves (2010), a partir de uma leitura analítica das portarias do SUS dos anos de 1998 a 2008, das 12.933 portarias encontradas, 51 fazem referência à Terapia Ocupacional, sendo 26 delas publicadas pelo Gabinete do Ministro (GM) e 25 pela Secretaria de Atenção à Saúde (SAS). Entretanto, tem-se que, dentre todas, somente 41 portarias estão vigentes atualmente e nenhuma disserta sobre a atuação em áreas nas quais a Terapia Ocupacional já se encontra inserida, como a área da oncologia, de queimados e da hanseníase.

Segundo Servantes (2002) o atendimento e tratamento de pacientes com câncer pela Terapia Ocupacional eram reconhecidos como medidas ou serviços de suporte, estabelecido pela Portaria $n^{\circ} 3.535$ da Política de Atenção Oncológica, parágrafo 3.5 , com data de 2 de setembro de 1998, emitida pelo Ministério da Saúde.

Macieira e Bifulco (2009) ao analisarem esta mesma portaria (MS/3.535/1998), verificaram a inclusão dos serviços de Terapia Ocupacional, e o de outras seis especialidades, como um importante critério para que haja o credenciamento das Unidades de Assistência de Alta Complexidade (UNACON) e dos Centros de Alta Complexidade em Oncologia (CACON) ${ }^{1}$. Assim, com relação à Terapia Ocupacional em Oncologia, no SUS, a categoria era prevista e colocada como um dos critérios mínimos para a existência dos CACON.

Neste cenário Servantes (2002) aponta a representação desta portaria como um expressivo marco para maior dimensão no campo de atuação e uma possibilidade de favorecer e melhorar o atendimento, tendo em vista a inclusão da profissão em equipe multiprofissional.

A Portaria $n^{\circ} 3.545 / 1998$ foi revogada em 8 Dezembro de 2005 e foi instituída a Política Nacional de Atenção Oncológica (Portaria no 2.439/GM de 8 de Dezembro de 2005) que contempla as ações de promoção, prevenção, diagnóstico, tratamento, reabilitação e cuidados paliativos a serem inseridas na rede de atenção oncológica no âmbito do SUS, afim de garantir qualidade ao atendimento, maior efetividade e eficiência no controle do câncer e diminuição do quadro de morbimortalidade (BRASIL, 2005).

As propostas da Política Nacional de Atenção Oncológica para gestão, formulação, execução e acompanhamento das ações em oncologia, almejam proporcionar aos pacientes o atendimento integral e humanizado. Asseguram a necessidade de múltiplas modalidades de tratamento em diferentes momentos da evolução da doença e apontam para o estabelecimento de serviços gerais, não oncológicos, como consultas em diversas especialidades (clínica médica, endocrinologia, pneumologia etc.), exames (laboratoriais, gráficos e

(1) De acordo com a Política Nacional de Atenção Oncológica, as UNACONs compreendem hospitais que possuem condições técnicas, instalações físicas, equipamentos e recursos humanos adequados à prestação de assistência especializada de alta complexidade para o diagnóstico definitivo e tratamento dos cânceres mais prevalentes no Brasil. Os CACONs são hospitais que possuem condições técnicas, instalações físicas, equipamentos e recursos humanos adequados à prestação de assistência especializada de alta complexidade para 0 diagnóstico definitivo e tratamento de todos os tipos de câncer. 
de imagem), suporte de outros profissionais da saúde, reabilitação e cuidados paliativos (BRASIL, 2010).

Cordeiro e Ioshimoto (2010) apontam que a nova legislação, não especifica a Terapia Ocupacional dentre as demais disciplinas de apoio multidisciplinar, como por exemplo, a Fisioterapia, Nutrição e Fonoaudiologia, ou seja, a Terapia Ocupacional ficou subentendida na definição de "reabilitação exigível conforme as respectivas especialidades".

\section{A prática terapêutica ocupacional no contexto hospitalar e junto à pessoa com câncer}

A assistência em Terapia Ocupacional contribui para a saúde dos indivíduos hospitalizados não levando em conta somente a doença ou a causa primária que a levou até o hospital, mas as repercussões psicológicas e sociais dessa situação. Por meio das atividades especificas e/ou exercícios selecionados de acordo com a individualidade do paciente e promovidos no próprio espaço hospitalar, o terapeuta ocupacional trabalhará todos os aspectos que influenciam de forma significativa a vida do paciente de modo a atender as reais necessidades.

Para Carlo et al. (2004, p. 9-10):

A nova perspectiva de assistência de Terapia Ocupacional no contexto hospitalar, que se volta para a importância de sua atuação como promotora da saúde e da qualidade de vida ocupacional mesmo durante o período de internação hospitalar, é bastante recente. Essa tendência norteia-se pelo principio da necessidade de manutenção não só da capacidade funcional, mas principalmente de um nível mais elevado de qualidade de vida, que implica maior auto-estima e melhores estados de humor e de motivação para a recuperação da saúde o mais rapidamente possível.

No que diz respeito aos indivíduos atingidos pelo câncer e a complexidade do adoecimento, o tratamento deve ser abrangente, merecendo atenção voltada as necessidades físicas, psicológicas e sociais; incluindo a participação da família, personalização da assistência, direito à informação e fornecimento de suporte social (ISHIBASHI, 2001).

De acordo com Othero (2010) o câncer é uma doença grave, com altas taxas de incidência e prevalência, e responsável por muitas mortes todos os anos. Portanto, é essencial que seja oferecido um serviço integrado capaz de contemplar todos os níveis de assistência em equipe multiprofissional.

Sendo assim, é neste contexto que se pode citar a importante participação do terapeuta ocupacional dentro do ambiente hospitalar para a assistência em Alta
Complexidade em Oncologia e atuando nos vários níveis de atenção à saúde, como a prevenção, promoção e recuperação da saúde, reabilitação, humanização do hospital e cuidados paliativos.

Em um levantamento com terapeutas ocupacionais, realizado pela Associação Brasileira de Linfoma e Leucemia sob a organização de Othero (2008) foram elencados os objetivos dos serviços prestados pela Terapia Ocupacional no campo da oncologia. Foram apontados: atenuar o impacto da doença e hospitalização; ajudar na elaboração do luto vivido pelo paciente e familiares; ajudar na adaptação das diferentes atividades cotidianas, a fim de resgatar a autonomia e independência; resgatar a autoestima e o equilíbrio emocional; oferecer medidas de conservação de energia; manter ou recuperar a capacidade funcional; prevenir incapacidades e deformidades osteomusculares; promover a reintegração social, a parceria entre paciente, família e cuidador e oferecer apoio emocional a todos os envolvidos no processo de adoecimento e tratamento (ABRALE, 2008).

Para Pengo e Santos (2004) referente à atuação do terapeuta ocupacional no hospital oncológico, o objetivo do trabalho é prestar atendimento ao paciente e familiares, desde a fase de diagnóstico, passando pelo processo ambulatorial ou de internação até o atendimento domiciliar. Ainda segundo essas autoras, há uma ampla diversidade da população atendida, abrangendo crianças, adolescentes e adultos, pós-transplantados de medula óssea, pós-cirúrgicos, pacientes em processo de quimioterapia e/ou radioterapia e em fase terminal.

Diante desses apontamentos, o objetivo principal do presente estudo foi identificar a inserção, atuação e ampliação do campo de trabalho da Terapia Ocupacional na área oncológica em contexto hospitalar, tomando por base a Política Pública de Atenção Oncológica vigente. Para tanto, foi realizada: identificação dos Centros de Alta Complexidade em Oncologia do Estado de São Paulo cadastrados no Cadastro Nacional de Estabelecimentos de Saúde, por meio de dados fornecidos pelo INCA e do site oficial do Ministério da Saúde; mapeamento da inserção do terapeuta ocupacional nesses locais e aplicação de questionário aos profissionais, para caracterizar os serviços e ações desenvolvidas pela Terapia Ocupacional em oncologia.

\section{MÉTODO}

Para alcance do objetivo proposto, foram selecionados todos os hospitais localizados no Estado de São Paulo classificados como CACON, que atendessem aos seguintes 
critérios de inclusão: fossem classificados como Centro CACON, estivessem devidamente cadastrados no Cadastro Nacional de Estabelecimentos de Saúde $(\mathrm{CNES})^{2}$ e que possuíssem o terapeuta ocupacional como parte da equipe multiprofissional para assistência de pacientes com câncer.

O questionário elaborado e enviado aos terapeutas ocupacionais, continha onze questões, a fim de caracterizar o perfil desses profissionais no que diz respeito: ao setor de atuação dentro do hospital; o tempo de atuação no setor e na área oncológica; o conhecimento e opinião desses profissionais referente ao papel das Políticas Públicas na atenção oncológica e com relação à inserção e atuação da Terapia Ocupacional e a importância da própria atuação junto aos pacientes com câncer dentro dos hospitais. O questionário também apresenta questões para caracterizar os hospitais, como anos de existência e a demanda de pacientes com câncer atendido pelo setor de Terapia Ocupacional.

\section{Procedimentos}

Para melhor compreensão do delineamento desta pesquisa, os procedimentos serão apresentados em etapas, a seguir:

Primeira etapa: foram reconhecidos todos os hospitais localizados no Estado de São Paulo classificados como CACON, segundo o Registro Hospitalar de Câncer fornecido pelo INCA em documento de junho de 2009.

Segunda etapa: de posse dessas informações, foi realizada busca dos CACONs, para selecionar apenas os que estavam devidamente cadastrados no CNES e que constavam a inserção de terapeutas ocupacionais como parte da equipe. Foi possível realizar esses levantamentos, pois no link do CNES no site oficial do Ministério da Saúde são disponibilizados os nomes de todos os hospitais cadastrados e dos profissionais empregados nos hospitais. O levantamento foi realizado no período de Março a Abril de 2011, por meio da internet na Biblioteca da UNESP campus de Marília.

Terceira etapa: os questionários foram enviados via correio, utilizando como referência o endereço dos hospitais e os nomes de todos os terapeutas ocupacionais selecionados. O período entre envio e recebimento da resposta foi de junho a setembro de 2011.
Junto ao questionário foi encaminhado uma carta e resumo do trabalho, para melhor informar os profissionais a respeito da pesquisa, e o Termo de Consentimento Livre e Esclarecido a ser assinado caso aceitassem participar do estudo.

O estudo seguiu preceitos éticos exigidos em pesquisas envolvendo seres humanos, apresentando parecer favorável do Comitê de Ética e Pesquisa da Faculdade de Medicina de Marília sob o n ${ }^{\circ}$ 577/11 em reunião ordinária de 27/06/11 e de acordo com a Resolução 196/96 do Conselho Nacional de Saúde.

Quarta etapa: foram escolhidos os participantes da pesquisa tendo como base a resposta enviada. Portanto estão incluídos nos resultados somente os profissionais que responderam ao questionário por completo.

Quinta etapa: realizada leitura e análise das respostas, de modo a organizar o conteúdo e discernir sobre a incorporação dos terapeutas ocupacionais no contexto das ações em oncologia hospitalar.

\section{RESULTADOS E DISCUSSÃO}

A partir do registro fornecido pelo INCA, 62 hospitais do Estado de São Paulo são classificados como CACON. Em pesquisa no site oficial do Ministério da Saúde identificou-se que 61 encontram-se cadastrados no CNES e, dentre todos, somente 43 possuem o terapeuta ocupacional como parte da equipe de profissionais.

Aos terapeutas ocupacionais destes 43 CACONs foi encaminhado o questionário. Destes CACONs, 10 fizeram contato, totalizando 25 terapeutas ocupacionais atuantes. Contudo apenas 10 dos 25 terapeutas ocupacionais responderam ao questionário; os outros 15 não atuam na área oncológica mesmo estando inseridos em contexto hospitalar e, por isso, optaram por não responder ao questionário, sendo desta forma, excluídos da pesquisa, assim como o hospital em que atuam.

Ao final desse processo de exclusão, participaram desta pesquisa 10 terapeutas ocupacionais distribuídos em seis hospitais. Os profissionais são denominados de sujeitos, portanto, sujeito 1 a sujeito 10; dos hospitais, três foram caracterizados como Hospitais Especializados (HE) de 1 a 3 e atendem somente pacientes com câncer, e três Santas

(2) O Cadastro Nacional de Estabelecimentos de Saúde (CNES) foi base para o conhecimento da realidade e das potencialidades da rede assistencial em saúde existente no território brasileiro e disponibilizou informações dos estabelecimentos que realizam ações e/ou serviços de saúde que estejam vinculados ao SUS e daqueles estabelecimentos de saúde ambulatoriais não vinculados ao SUS. Essas informações caracterizaram as atuais condições desses locais nos aspectos de Área Física, Recursos Humanos, Equipamentos e Serviços Ambulatoriais e Hospitalares. 
Casas caracterizadas como SC de 1 a 3. Esta denominação é para preservar o nome dos sujeitos e dos hospitais aqui apresentados.

Vale ressalvar que os sujeitos 1,2 e 3 atuam no mesmo hospital (HE 3), assim como os sujeitos 6, 7 e 8 que também trabalham no mesmo local (HE 1); os demais participantes estão inseridos em locais distintos (HE 2, SC 1, SC 2 e SC 3).

A seguir a apresentação dos dados será ilustrada a partir das perguntas contidas no questionário respondido pelos terapeutas ocupacionais.

Em relação aos anos de existência do Hospital onde trabalha, observou-se que a maioria desses locais são antigos, possuindo mais de 80 anos de existência; com exceção dos hospitais especializados 1 e 2 que possuem menos de 20 anos. $\mathrm{O}$ tempo de existência destes hospitais está demonstrado na Figura 1.

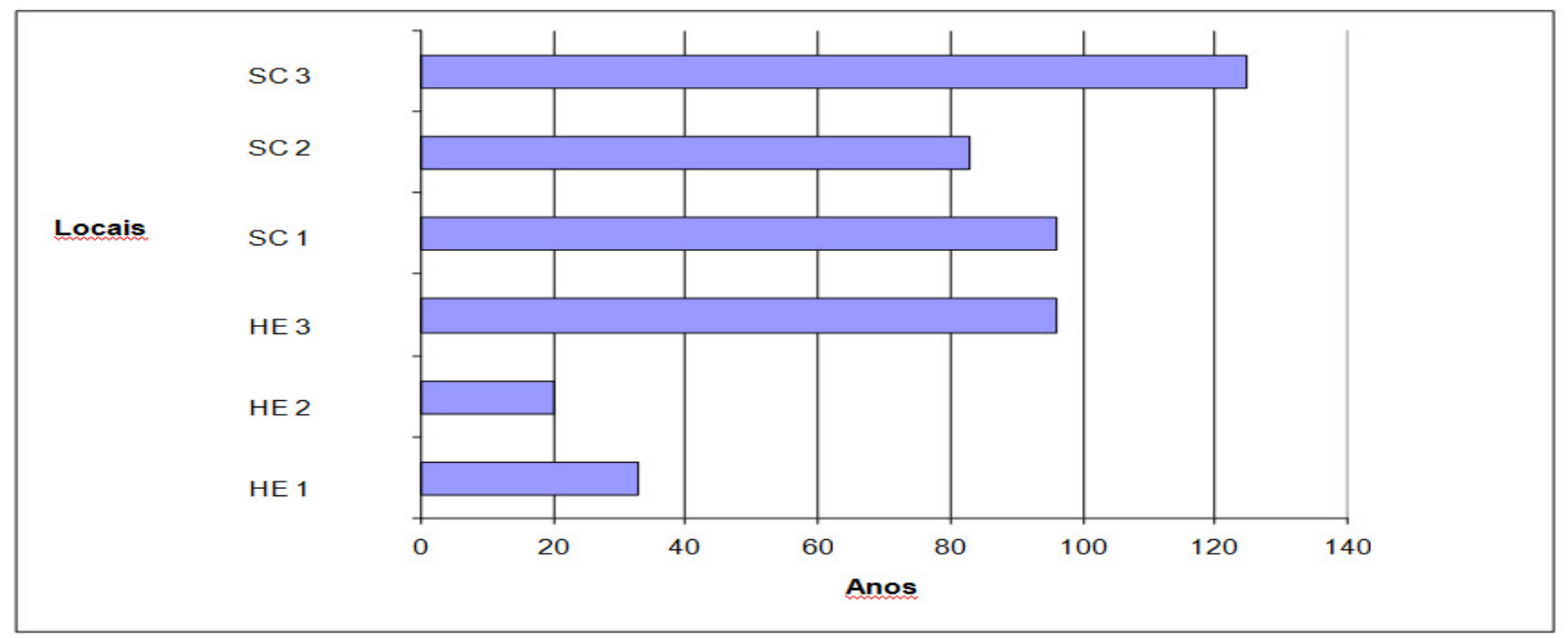

Figura 1 - Anos de existência dos hospitais

Quanto ao tempo de atuação dos terapeutas ocupacionais na oncologia, nove profissionais atuam há menos de 4 anos e apenas um atua há 27 anos. Sendo o tempo mínimo de um ano e o tempo máximo de 27 anos, conforme demonstrado na Figura 2.

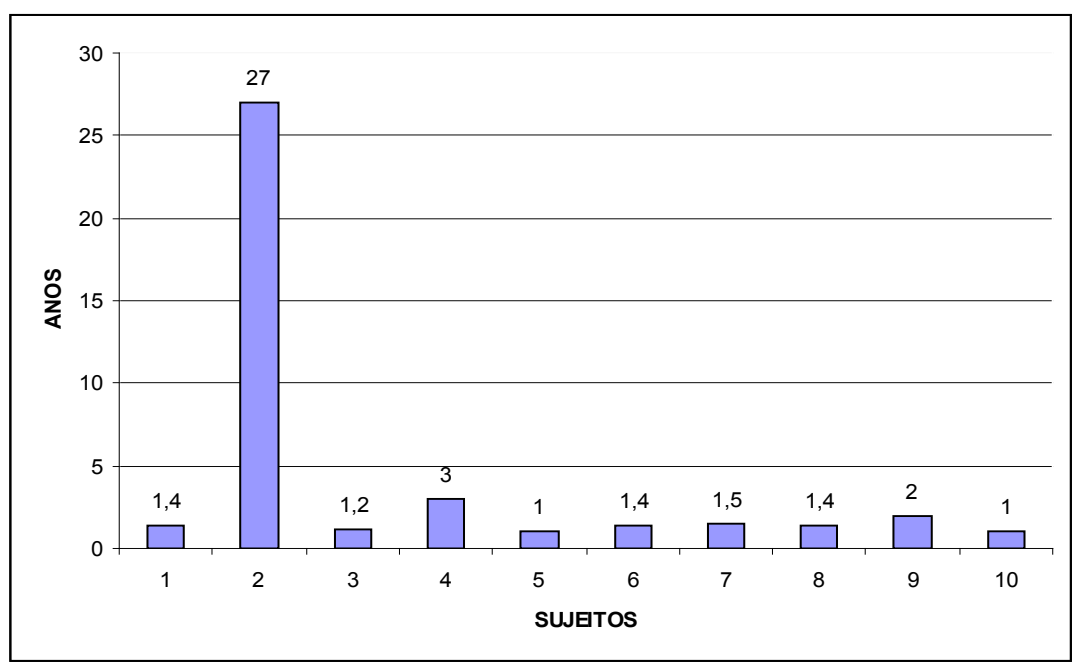

Figura 2 - Tempo de atuação dos terapeutas ocupacionais na área oncológica 
A identificação do tempo de existência dos hospitais e de atuação dos terapeutas ocupacionais em oncologia nestes locais se faz importante quando se destaca a promulgação da nova Política Nacional de Atenção Oncológica (Portaria no 2.439/GM de 8 de dezembro de 2005), que ocorreu há seis anos, ou seja, a atenção para indivíduos com câncer está em expansão desde a publicação desta legislação. Assim como se encontra em recente expansão a incorporação da Terapia Ocupacional nesta área de atuação, caracterizando-a com certo atraso, uma vez que, mesmo estando revogada a Portaria $n^{\circ} 3.535 / 98$ para atenção oncológica, há 12 anos já abordava a inserção da profissão como um serviço de suporte e um dos critérios mínimos para existência dos CACON.

$\mathrm{Na}$ caracterização dos setores onde esses profissionais trabalham, verificou-se que a maioria (sete profissionais) realiza os atendimentos em ambulatório de oncologia adulto, contudo, também estão incorporados em outros setores como: enfermaria e ambulatório infantil, enfermaria adulto, brinquedoteca, unidade de transplante de medula óssea e unidade de terapia intensiva. Esses dados estão demonstrados na Figura 3.

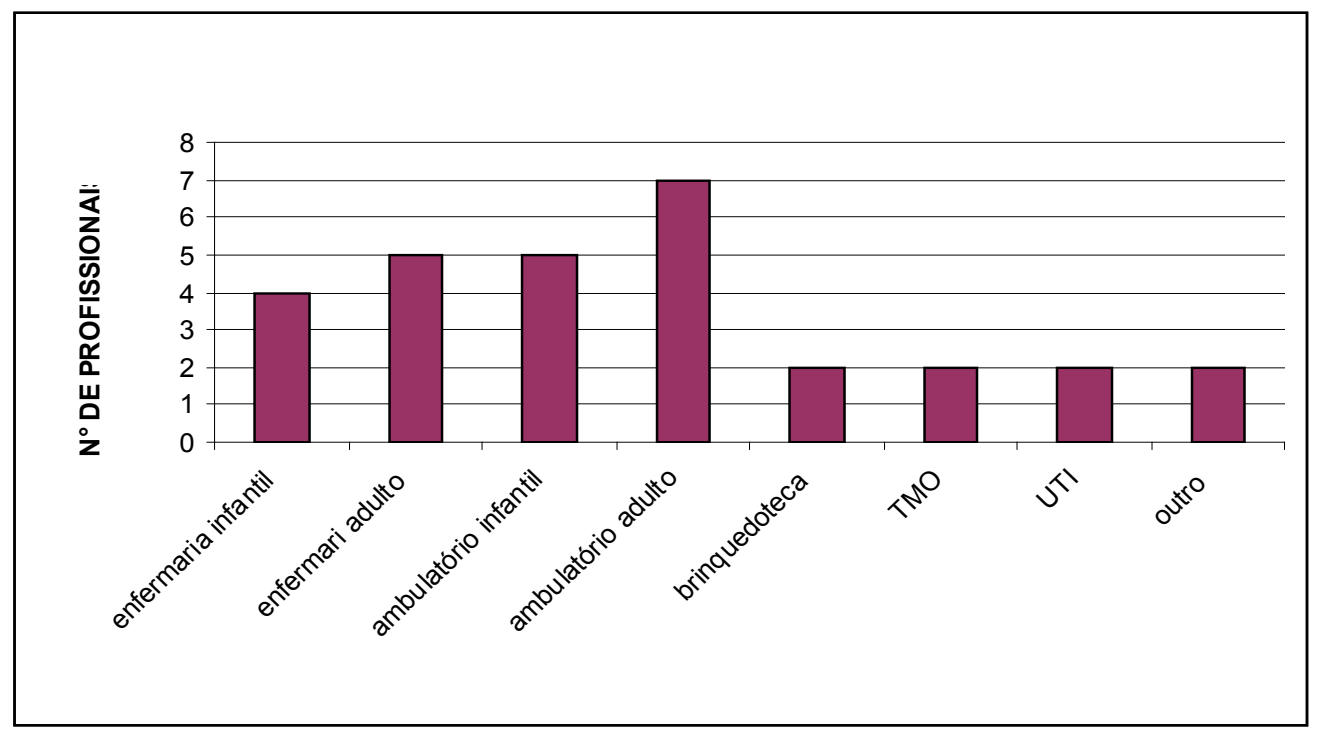

Figura 3 - Setores de trabalho dentro do hospital

Dois profissionais destacaram outros locais de atuação como o setor de mastologia, direcionado aos atendimentos às mulheres com câncer de mama que necessitam de orientações adequadas para iniciar as atividades cotidianas no pós-cirúrgico e, a sala de apoio pedagógico onde são realizadas ações em conjunto com a pedagogia, fonoaudiologia e com a própria escola (quando necessário), voltadas a viabilização das atividades escolares das crianças e jovens hospitalizados.

Sobre a realização de visitas domiciliares, somente dois profissionais ( $\mathrm{S} 2$ e $\mathrm{S} 3$ ) relataram realizar essa prática. Ambos trabalham no mesmo hospital (H3) e atendem pacientes que já receberam alta e residem no mesmo município onde está localizado o hospital. Os pacientes além de receberem visita dos terapeutas ocupacionais, também são assistidos pelo serviço social, fisioterapeutas, psicólogos, enfermeiros e médicos.

Não foram encontradas referências na Política Nacional de Atenção Oncológica sobre a assistência voltada à visita domiciliar. Contudo, segundo Rocha et al. (2010), sob a perspectiva da integralidade do cuidado à saúde dos pacientes oncológicos, deve-se oferecer o atendimento domiciliar após a alta hospitalar.

Para Bigatão et al. (2010) um dos objetivos que norteiam a prática da Terapia Ocupacional na visita domiciliar é auxiliar o paciente e sua família na reorganização da rotina, de modo a promover a saúde por meio de orientações e intervenções pontuais e, consequentemente, evitar as internações recorrentes e gastos.

Como abordado anteriormente, a nova Política Nacional de Atenção Oncológica não aponta claramente as atribuições do terapeuta ocupacional, mas a partir dos relatos dos profissionais entrevistados, nota-se uma variedade de ações e serviços oferecidos e os diferentes setores onde podem se inserir, iniciando uma representativa mais expressiva da profissão.

A respeito da demanda de pacientes atendida pelos terapeutas ocupacionais, observa-se que esta é diversificada 
quanto: à quantidade de pacientes, aos tipos de câncer e a faixa etária atendida. Em um dos hospitais (HE 3) os terapeutas ocupacionais atendem em média 350 pacientes/ mês. É importante considerar que o número elevado de pacientes atendidos nesse HE é justificado por sem um centro de referência, prestando atendimento à população de outros municípios da região. Os outros hospitais atendem em média de 40 a 100 pacientes/mês.

Os tipos de câncer destacados como sendo de maior prevalência nesses hospitais foram: leucemia, tumores ósseos, tumores de partes moles, do sistema nervoso central e câncer de mama. Contudo, há uma gama variada de tipos de câncer atendidos segundo os profissionais.

Em relação à idade dos pacientes atendidos, a faixa etária compreende desde o nascimento até os 80 anos de idade, sendo que a maioria dos profissionais atende a população jovem adulta. Isso permite concluir a possibilidade de se trabalhar com pessoas de todas as idades, desde a infância até a terceira idade.

A esses registros, tanto da demanda de pacientes com câncer, assim como da faixa etária dos mesmos, pode-se destacar que a incidência de câncer no Brasil vem crescendo a cada ano, levando em consideração o envelhecimento populacional e os estilos e hábitos de vida não saudáveis. No Brasil as estimativas para o ano de 2010, também válidas para 2011, indicaram a ocorrência de 489.270 casos novos de câncer (BRASIL, 2009).

Quando questionados sobre o papel das Políticas Públicas na atenção oncológica todos os profissionais apontam para a garantia de uma melhor assistência, ou seja, que as Políticas específicas dessa área vêm crescendo a cada ano, a fim de se efetivar as ações e serviços no controle e prevenção do câncer, na promoção de saúde, diagnóstico, tratamento e reabilitação. Desta forma, se qualifica o atendimento e se ganha o reconhecimento de todos os profissionais e usuários do SUS. Ainda assim, o sujeito 7 salienta a necessidade de implementação das Políticas Públicas de acordo com a realidade de cada região, para atender as reais demandas e suprir as necessidades de recursos humanos, materiais e sociais.

Sobre essa questão o sujeito 9 aponta que:

"no papel a política é bem elaborada, mas na prática verifica-se dificuldades em atender pacientes com medicamentos especificos que não estão no 'rol'do SUS, tendo que conseguir seu direito via judicial".

A respeito do conhecimento dos terapeutas ocupacionais sobre a Política Nacional de Atenção Oncológica com relação à inserção e atuação da Terapia
Ocupacional nessa área de atendimento, conclui-se que a profissão começa a ganhar espaço, porém não se tem esclarecido o tipo de atuação e as Políticas Públicas desconhecem a importância dos serviços oferecidos pela Terapia Ocupacional.

Para os sujeitos 4 e 9 as Políticas ainda são incipientes e não relatam as atribuições da Terapia Ocupacional, sendo necessário cada terapeuta ocupacional se encaixar na demanda e necessidade do setor oncológico. Somado a isso, os sujeitos 2, 7, 8 e 9 relatam que para alcançar o sucesso na inserção e no reconhecimento da profissão pelas Políticas Públicas, profissionais e pacientes só é possível por meio do trabalho realizado e, desta forma, mostrar para toda equipe e pacientes o quão válida é a atuação da Terapia Ocupacional.

Para o sujeito 5 a Terapia Ocupacional é rara no mercado de trabalho como se pode constatar em seu relato:

"são poucos os demais profissionais que conhecem nossa profissão e esse desconhecimento causa a fragilidade de nossa ação" e ainda acrescenta que "a permanência depende das ações desenvolvidas, cursos e capacitação dos profissionais".

Conclui-se, portanto, que a profissão está começando a ganhar espaço, porém as Políticas Públicas em Oncologia desconhecem a importância dos serviços oferecidos pela Terapia Ocupacional ao deixarem de mencionar esta categoria de forma clara e objetiva. Segundo Beirão e Alves (2010) as portarias na SAS e GM não fazem referência e, tão pouco, regulamentam a atuação da Terapia Ocupacional na área oncológica. E diante da falta de atributos que norteiam a prática, torna-se difícil estruturar um serviço e trabalhar nesse campo.

Especificamente em relação às abordagens terapêuticas, os sujeitos 1 e 3 salientam que a abordagem terapêutica ocupacional se faz importante na medida em que se busca trabalhar com o lado saudável do paciente, mostrando as possibilidades de viver com qualidade mesmo diante da doença e hospitalização e minimizando as consequências negativas. De acordo com o sujeito 7 por meio dos recursos terapêuticos utilizados no processo de tratamento é possível observar e identificar as dificuldades do paciente, assim como desenvolver as habilidades e explorar possibilidades e potencialidades.

Para os sujeitos 2 e 4 é o terapeuta ocupacional responsável por reestruturar as atividades significativas dos pacientes, uma vez que o processo de adoecimento e hospitalização desencadeiam rupturas no cotidiano. Somado a isso, pode-se acrescentar os relatos dos sujeitos 5 e 6 que apontam para a importância da atuação, por meio da 
reabilitação física e treino de atividades de vida diária e de vida prática, quando se almeja retomar as atividades e/ou buscar novos afazeres e manter o desempenho ocupacional.

Mediante a possibilidade dos pacientes continuarem a desempenhar seus papéis perante a sociedade, cria-se um espaço para a reinserção social e também uma oportunidade de mostrar o valor da Terapia Ocupacional, já que em seus processos aborda várias dimensões do sujeito, inclusive a rede social na qual o mesmo se relaciona.

O sujeito 7 aborda a atuação em diferentes níveis, em busca da autonomia e independência dos pacientes, tais como:

"orientação e suporte ao familiar/cuidador, reabilitação física e cognitiva do paciente, sendo o foco do trabalho direcionado pelas necessidades do individuo doente e pelo setor de atuação do terapeuta ocupacional".

Já o sujeito 8 diz que a importância se encontra na humanização hospitalar, melhorando a qualidade de vida durante a hospitalização.

Sendo objetivo primordial da Terapia Ocupacional promover a qualidade de vida, a humanização abrange diversas estratégias que atingem o ambiente hospitalar, os pacientes, os familiares, a equipe de saúde e demais funcionários.

O sujeito 9 acredita no potencial da profissão em contexto hospitalar na atenção oncológica,já que podem e devem oferecer o suporte necessário no processo de alta hospitalar. E acrescenta:

"quando o paciente é bem orientado, nós conseguimos diminuir a quantidade de retorno nos ambulatórios e deixamos vagas para novos casos, gerando assim um aumento no fluxo de atendimento na oncologia”

O sujeito 10 oferece destaque para a situação psicológica dos pacientes e aborda a importância de atuar em conjunto com a família no processo de aceitação da doença.

Hoje, o câncer é reconhecido como um problema de saúde pública que requer abordagem multidisciplinar e interdisciplinar, a fim de realizar diagnósticos precoces e cuidar da prevenção do câncer e promoção da saúde, reduzir a mortalidade e melhorar a qualidade de vida do paciente (D'ANGELO, 2007). Desta forma, a partir dos levantamentos e de acordo com D'Angelo (2007), verificase que todos os profissionais entrevistados apontam para diferentes aspectos da atuação terapêutica ocupacional, classificando-a como uma modalidade de suporte importante e habilitada para atender a demanda oncológica.

\section{CONCLUSÃO}

Quando o individuo é acometido pelo câncer criase uma situação de vulnerabilidade, pois, além dos fatores desencadeantes da própria doença, existem problemas psicossociais preexistentes ao câncer. Portanto, é preciso que o tratamento ultrapasse o limite do cuidado biológico e se intere do modelo de assistência integral de modo a construir uma abordagem holística e interdisciplinar.

Para que se cumpra o paradigma desse modelo, é inerente ao campo da saúde possuir vários campos de atuação. Assim, os profissionais que compõem uma equipe, todos complementares e interdependentes, somam esforços, a fim de se obter o êxito total da cura, visto como a melhora dos aspectos biológicos, sociais e psicológicos, assim como proporcionar o conforto necessário àqueles que estão fora das possibilidades de cura e, portanto, em fase paliativa do tratamento.

Os benefícios são significativos e todos são recompensados. Os profissionais têm satisfação pelo trabalho realizado e pelo reconhecimento, os pacientes e familiares alcançam o que desejam: serem tratados com dignidade, respeito à vida e direito ao tratamento com qualidade.

A essas colocações somam-se os apontamentos de Moribe e Massumoto (2009), que destacam o terapeuta ocupacional como apto a compor uma equipe multidisciplinar, para dar o suporte necessário aos pacientes em tratamento oncológico e aos familiares envolvidos nesse processo.

Neste presente estudo foi possível identificar a inserção, atuação e ampliação do campo de trabalho da Terapia Ocupacional na área oncológica, porém com limitações, visto tal investigação ter sido feita somente no Estado de São Paulo e pelo pouco retorno dos profissionais.

Quanto à inserção, foi possível verificar que nem todos os hospitais pesquisados contam com os serviços e ações da Terapia Ocupacional e que a chegada dos profissionais a esses setores é recente, tendo em vista os anos de atuação identificados. Além disso, no mapeamento para a identificação dos CACONs, pôde-se verificar que alguns municípios de pequeno porte ou mesmo os menores hospitais não possuem o terapeuta ocupacional na gama de profissionais. A partir disso fica o questionamento: será que esses fatores justificam a ausência desses profissionais? Ou será que os profissionais, quando formados, escolhem os grandes centros em detrimento de melhores oportunidades 
e/ou salários?

Ainda assim podemos assinalar a formação dos terapeutas ocupacionais, uma vez que nem todos os graduandos têm a oportunidade de atuar nesse campo, quando em estágio curricular. Segundo o sujeito 5, sua graduação não lhe ofereceu tal possibilidade, criando dificuldade ao iniciar trabalho no hospital.

Para De Carlo e Luzo (2004) a Terapia Ocupacional vem ganhando reconhecimento profissional e social na área hospitalar, mas para que a inserção seja efetivada com sucesso e se criem novas possibilidades, é preciso colocar em pauta a experiência e conhecimento profissional, as necessidades e possibilidades da clientela atendida e as características do hospital (capacidade física, quais as ações e serviços prestados e a demanda de pacientes).

Considerando a inexistência de portarias específicas na Política Nacional de Atenção Oncológica relacionadas às competências do terapeuta ocupacional dentro dos CACONs, identificou-se para o campo da atuação, a existência de diversas possibilidades práticas. Cada profissional atua seguindo um referencial teórico-metodológico, e constrói o planejamento terapêutico de acordo com as necessidades e problemáticas apresentadas pela população atendida, com o espaço hospitalar, os serviços oferecidos e recursos materiais disponíveis.

O fato da Terapia Ocupacional não ser mencionada de forma específica na legislação atual, revela um fato de preocupação para a área, principalmente para a ampliação e consolidação da profissão no campo da assistência de Alta Complexidade em Oncologia, já que leis, normas e portarias favorecem uma atuação adequada dentro da organização assistencial do sistema de saúde e oferecem um suporte para consolidação das ações e serviços.

Como exemplo, tem-se o resultado da implantação de políticas públicas em saúde mental que, somada aos esforços dos próprios profissionais, possibilitou a incorporação de cerca de 200 terapeutas ocupacionais no contexto das ações em saúde mental; ilustrando a importância da discussão e criação de Política Pública para as práticas assistenciais e mercado de trabalho (OLIVER et al., 2005).

Diante desses achados e tomando por base os apontamentos de Cavalcanti e Galvão (2007), para que se criem novas possibilidades de trabalho e reconhecimento se faz necessário que o terapeuta ocupacional se posicione diante da equipe e da comunidade, de modo a esclarecer as competências e habilidades desta profissão.

Por fim, neste contexto se ressalta a importante contribuição desta pesquisa, por oferecer uma base teórica que intensifica os conhecimentos quanto ao tema proposto e, consequentemente, auxilia para o envolvimento efetivo desses profissionais no processo de gestão, organização e monitoramento do próprio serviço oferecido, mediante as Políticas Públicas em saúde e às práticas clínicas. Além disso, instiga a importância e necessidade de se investir em novas pesquisas que possam contribuir para evolução e ampliação da Terapia Ocupacional na área oncológica.

\section{ANEXO 1 - Questionário}

1. Nome (opcional):

2. Nome e anos de existência do Hospital/Centro onde trabalha:

3. Há quanto tempo trabalha neste Hospital/Centro como terapeuta ocupacional?

4. Há quanto tempo trabalha na área oncológica no contexto hospitalar?

5. Quais ou qual seu local de trabalho dentro do hospital?

( ) enfermaria infantil ( ) enfermaria adulto

( ) ambulatório de pediatria ( ) ambulatório adulto

( ) brinquedoteca ( ) setor de TMO

()UTI outro:

6. Qual a demanda de pacientes com câncer atendida pela Terapia Ocupacional?

7. Qual a faixa etária atendida?

8. São realizadas visitas domiciliares aos pacientes quando recebem alta?

( ) $\operatorname{sim}$ ( ) não. Se sim, por quais profissionais?

9. Em sua opinião, qual é o papel das Políticas Públicas na atenção oncológica?

10. Em sua opinião, qual é o papel das Políticas Públicas na atenção oncológica com relação à inserção e atuação da Terapia Ocupacional nesta área de tratamento?

11. Em sua opinião, qual é a importância da atuação do terapeuta ocupacional junto aos pacientes com câncer dentro dos hospitais/centros? 12. Deixe aqui, comentários, sugestões ou reflexões: 
SILVA, A. C. C.; GIUARDINETTO, A. R. S. B. Public policies in oncology: reflecting on the role of occupational therapy. Rev. Ter. Ocup. Univ. São Paulo, v. 23, n. 3, p. 297-308, set./dez. 2012.

\begin{abstract}
The National Policy Oncological Care aims access and whole care and a greater effectiveness and efficiency in cancer control. Thus, several actions, and professional services are required to provide the necessary assistance. Regarding the role of occupational therapy, it is noted that the profession is not specified, and the action has not regulated. Therefore, the main objective of this study was to identify the insertion, acting and expansion of the field work of Occupational Therapy in the State of São Paulo for the oncology hospital in context, based on the Public Policy of Oncology Care. Also aimed to identify the Service Centers of High Complexity in Oncology of the State of São Paulo who possess the occupational therapist as part of the team. Therefore, it was held: identification of Centers of High Complexity Oncology of São Paulo registered in the National Register of Health Care Facilities, using data provided by INCA and the official website of the Ministry of Health; mapping the inserting of an Occupational Therapist in these locations and aplication of questionnaire to professionals, to characterize the services and actions developed by occupational therapy in oncology. The descriptive qualitative study, found that there are different possibilities of care in oncology, in view of the demands and organization of hospitals. As for the insertion and expansion of the field work, it was found that not all CACONs have the services and activities of Occupational Therapy, which suggests that there is not effectively incorporate this professional in multidisciplinary teams in these centers.
\end{abstract}

KEYWORDS: Oncology/manpower; Health public policy; Occupational therapy; Oncology service, hospital/manpower

\title{
REFERÊNCIAS
}

ASSOCIAÇÃO BRASILEIRA DE LINFOMA E LEUCEMIA (ABRALE). Terapia ocupacional na oncologia. In: OTHERO, M. B.(Org.). Comitê de Terapia Ocupacional de Associação Brasileira de Linfoma e Leucemia. 2008. Disponível em: <http://www.abrale. org.br/arquivos/TERAPIA \%20OCUPACIONAL\%20NA\%20 ONCOLOGIA.doc $>$. Acesso em: 22 de mar de 2011.

BEIRÃO, R. O. S.; ALVES, C. K. A. Terapia ocupacional no SUS: refletindo sobre a normatização vigente. Cad. Ter. Ocup. UFSCar, v. 18 , n. 3, p.231-246, 2010.

BIGATÃO, M. R.; MASTROPIETRO, A. P.; CARLO, M. M. R. P. Terapia ocupacional em oncologia - a experiência do hospital das clínicas da Faculdade de Medicina de Ribeirão Preto da Universidade de São Paulo. In: OTHERO, M. B. Terapia ocupacional práticas em oncologia. São Paulo: Roca, 2010. p. 302-322.

BRASIL. Instituto Nacional do Câncer. Estimativa 2010: incidência de câncer no Brasil. 2009. Disponível em: $<$ http:// www.inca.gov.br/estimativa/2010/index.asp?link=conteudo_view. asp \&ID=5> . Acesso em: 25 de mar. 2011.

BRASIL. Ministério da Saúde. Entendendo o SUS. Disponível em: <http://portal.saude.gov.br/portal/arquivos/pdf/cartilha entendendo_o_sus_2007.pdf $>$. Acesso em 26 de jan. 2011.
BRASIL. Ministério da Saúde. Política Nacional de Atenção Oncológica. Brasília, 2010. <Disponível em: http://portal.saude. gov.br/portal/arquivos/pdf/infodae_oncologia.pdf.>. Acesso em 3 de fev. 2011.

BRASIL. Ministério da Saúde. Portaria $\mathbf{n}^{\circ}$ 2439/GM, de 8/ 12/2005. Política Nacional de Atenção Oncológica. Brasília, 2005. 3p.

BRASIL. Ministério da Saúde. O que é o SUS. Brasília. Disponível em: $<$ http://portal.saude.gov.br/portal/saude/cidadao/area.cfm?id area $=1395>$. Acesso em 12 de jan. 2011.

BRASIL. Ministério da Saúde. A política de saúde no Brasil nos anos 90: avanços e limites. Brasília, 2002. 52p.

CARLO, M. M. R. P.; BARTALOTTI, C. C.; PALM, R. D. C. M. A terapia ocupacional em reabilitação física e contextos hospitalares: fundamentos para pratica. In: CARLO, M. M. R. P.; LUZO, M. C. M. Terapia ocupacional: reabilitação física e contextos hospitalares. São Paulo: Roca, 2004. p. 3-28.

DE CARLO, M. M. R. P.; LUZO, M. C. M. Considerações finais. In: DE CARLO, M. M. R. P.; LUZO, M. C. M. Terapia ocupacional: reabilitação física e contextos hospitalares. São Paulo: Roca, 2004. p. 305-308. 
CAVALCANTI, A.; GALVÃO, C. Trabalho em equipe. In: CAVALCANTI, A.; GALVÃO, C. Terapia ocupacional. fundamentação e prática. Rio de Janeiro: Guanabara Koogan, 2007. p. 35-37.

CORDEIRO, J. J. R.; IOSHIMOTO, M. T. A. Organização de serviços de terapia ocupacional - gestão a partir de dados e indicadores. In: OTHERO, M. B. Terapia ocupacional práticas em oncologia. São Paulo: Roca, 2010.

ISHIBASHI, A. The needs of children and adolescents with cancer for information and social support. Cancer Nurs, New York, v. 24, n. 1, p. 61-67, 2001.

MACIEIRA, R. C.; BIFULCO, V. A. Formação para a prática da interdisciplinaridade. In: VEIT. M. T. Transdisciplinaridade em oncologia: caminhos para um atendimento integrado. São Paulo: HR, 2009. p.127-132.

MORIBE, I.; MASSUMOTO, C. Atendimento integral ao paciente portador de câncer e o terceiro setor. In: VEIT. M. T. Transdisciplinaridade em oncologia: caminhos para um atendimento integrado. São Paulo: HR, 2009. p. 142-144.

OTHERO, M. B. Terapia ocupacional práticas em oncologia. São Paulo: Roca, 2010.

OLIVER, F. C.; BARROS, D. D.; LOPES, R. E. Estudo sobre a incorporação da terapia ocupacional no contexto das ações de saúde mental e saúde da pessoa com deficiência no Municipio de São Paulo entre 1989 e 1993. Rev. Ter. Ocup. Univ. São Paulo, v. 16, n. 1, p. $31-39,2005$.

PENGO, M. M. S. B.; SANTOS, W. A. O papel do terapeuta ocupacional em oncologia. In: CARLO, M. M. R. P.; LUZO, M. C. M. Terapia ocupacional: reabilitação física e contextos hospitalares. São Paulo: Roca, 2004. p.233-255.

ROCHA, C. R.; et al. Terapia ocupacional em assistência oncológica domiciliar - a experiência de São Paulo internações domiciliares. In: OTHERO, M. B. Terapia ocupacional práticas em oncologia. São Paulo: Roca, 2010. p. 365-387.

SERVANTES; L. F. Terapia ocupacional: pesquisa e atuação em oncologia. Campo Grande: UCDB, 2002.

Recebido para publicação: 27/01/2012

Aceito para publicação: 05/08/2012 
SILVA, A. C. C; GIUARDINETTO, A. R. S. B. Politicas. Rev. Ter. Ocup. Univ. São Paulo, v. 23, n. 3, p. $297-308$, set./dez. 2012. 